\section{Sehr geehrte Mitglieder,}

am 23. Oktober 2020 fand die erste OnlineMitgliederversammlung der DEGUM statt. Der Vorstand hatte sich im Sommer für dieses Format entschieden, weil die Entwicklung der Pandemie zu jenem Zeitpunkt nicht abzusehen war. Im Nachhinein muss man vor dem Hintergrund der derzeitigen Situation zu dem Schluss kommen, dass diese Entscheidung die einzig richtige war. Auch wenn sich der Start der Veranstaltung aufgrund technischer Ungereimtheiten (es war eben eine Premiere) etwas verzögerte, so gestaltete sich der weitere Ablauf doch unproblematisch. Im Rahmen der Versammlung wurde turnusgemäß der neue Vorstand gewählt. Er setzt sich für die kommenden Jahre wie folgt zusammen:

Präsident: Prof. Dr. med. Josef Menzel, Sektion Innere Medizin

Vizepräsident: Prof. Dr. med. Peter Jecker, Sektion Kopf-Hals

Neupräsident: Prof. Dr. med. Markus Hahn, AK Mamasonografie

Sekretär: Prof. Dr. med. Hans Heynemann, Sektion Urologie

Schatzmeister: Prof. Dr. med. Christoph Berg, Sektion Gynäkologie und Geburtshilfe Beisitzer: Dr. med. Karsten Pracht, Sektion Anästhesie

Beisitzer: Dr. med. Sabine Guth, Sektion Innere Medizin

Aus dem Vorstand sind PD Dr. med. Kai Heling und Prof. Dr. med. Peter Kozlowski (beide aus der Sektion Gynäkologie und Geburtshilfe) sowie Dr. med. Siegfried Krishnabhakdi, Sektion Chirurgie, ausgeschieden. Alle 3 wurden im Herbst 2014 im Rahmen des DLT in Innsbruck in den Vorstand gewählt. In dem 6-jährigen Zeitraum haben sie die Gesellschaft durch ihren unermüdlichen Einsatz entscheidend mitgeprägt. Deshalb möchten wir den Dreien im Namen aller Mitglieder der DEGUM einen ganz besonderen Dank aussprechen.

Jetzt gilt es, in neuer Zusammensetzung die Geschicke der DEGUM in die Hand zu nehmen, und das in Zeiten, die - wie Sie alle wissen - nicht einfach sind. Eine Gesellschaft wie die DEGUM lebt vom Wandel. Durch die Neuzusammensetzung des Vorstands ergeben sich neue Ideen und Möglichkeiten, die der Gesellschaft gut tun werden. Wir dürfen alle auf die zukünftige Entwicklung gespannt sein.

Mit Prof. Dr. med. Christoph Berg konnte eine Persönlichkeit gewonnen werden, die seit langem in der DEGUM in verschiedenen Gremien aktiv tätig ist. Er ist Stufe-III-Kursleiter der DEGUM, war langjähriger Leiter des Arbeitskreises Fetale Echokardiografie der DEGUM, Mitglied des Educational Committee der International Society for Ultrasound in Obstetrics and Gynecology (ISUOG) und ist Vizepräsident der World Association of Perinatal Medicine (WAPM). Prof. Dr. med. Christoph Berg ist gleichzeitig Leiter des Bereichs für Pränatale Medizin und Gynäkologische Sonografie der Universitätsfrauenklinik Köln, Leiter des Bereichs Fetalchirurgie der Universitätsfrauenklinik Bonn und zusätzlich am Marienhospital in Witten tätig.

Mit Dr. Karsten Pracht bekleidet zum ersten Mal ein Vertreter aus der Sektion Anästhesie eine Vorstandsposition. Innerhalb weniger Jahre ist die Anästhesie von 600 Mitgliedern auf 1200 Mitglieder zur drittgrößten Sektion innerhalb der DEGUM angewachsen.
Dr. Karsten Pracht (DEGUM Stufe II) ist seit 2004 Chefarzt der Klinik für Anästhesie, Intensivmedizin, Schmerztherapie und Palliativmedizin der Sana-Kliniken Leipziger Land. Seit 2009 entwickelt Herr Dr. Pracht strukturierte Ausbildungskonzepte und diskutiert diese auf nationalen und internationalen Kongressen. 2016 übernahm er die Leitung der Sektion Anästhesiologie und engagierte sich in der inhaltlichen Weiterentwicklung der Sektion, wie Aktualisierung der Ausbildungscurricula sowie der Neueinführung von Curricula für Echokardiografie in der Anästhesie und Intensivmedizin und Ultraschall in der Behandlung chronischer Schmerzpatienten.

Als weiteres Mitglied wurde Dr. Sabine Guth in den Vorstand gewählt. Der Schwerpunkt ihrer Arbeit ist die internistische sonografische Früherkennung im Rahmen von Vorsorgeuntersuchungen. Sie ist seit 2005 Ausbilderin der DEGUM, seit 2007 hat sie die Stufe III inne und ist seit 2017 Kursleiterin. Dr. Sabine Guth arbeitete viele Jahre im Universitätsklinikum HamburgEppendorf, davon 7 Jahre durchgehend in der Medizinischen Sonografie, deren stellvertretende Leiterin sie war, bis sie 2006 die Leitung der Ultraschallabteilung des Medizinischen Präventions Centrums Hamburg am UKE übernahm (jetzt Conradia Medical Prävention Hamburg).

Prof. Dr. med. J. Menzel

Präsident der DEGUM

Prof. Dr. med. P. Jecker

Vizepräsident der DEGUM 